\title{
Hypersensitivity and infusion-site adverse events with intravenous fosaprepitant after anthracycline-containing chemotherapy: a retrospective study
}

\author{
Ralph Boccia*,1, Robert B Geller², Neil Clendeninn ${ }^{\ddagger 2} 2$ \& Thomas Ottoboni ${ }^{2}$ \\ ${ }^{1}$ Center for Cancer \& Blood Disorders, 6410 Rockledge Drive, \#660, Bethesda, MD 20817, USA \\ ${ }^{2}$ Heron Therapeutics, Inc., 4242 Campus Point Court, Suite 200, San Diego, CA 92121, USA \\ *Author for correspondence: Tel.: +1 301580 4882; rboccia@ccbdmd.com \\ ¥Employee at time of study, currently consultant to Heron Therapeutics, Inc
}

\begin{abstract}
Aim: Fosaprepitant, an intravenous neurokinin-1 receptor antagonist for chemotherapy-induced nausea and vomiting, contains polysorbate 80 , which is associated with infusion-site adverse events (ISAEs) and hypersensitivity systemic reactions (HSRs). This study investigated ISAEs/HSRs following fosaprepitant with anthracycline-containing chemotherapy. Patients \& methods: This retrospective chart review noted ISAEs/HSRs following the anthracycline doxorubicin+cyclophosphamide and a three-drug fosaprepitant regimen, via peripheral line. Results: $35 / 127$ patients (28\%) developed ISAEs/HSRs with chemotherapy and antiemetic therapy: 32 developed 137 individual ISAEs, primarily erythema, pain and catheter-site swelling; 16 developed 50 individual HSRs, primarily edema/swelling, erythema or dermatitis (no anaphylaxis). Conclusion: Fosaprepitant is associated with a significant ISAE/HSR rate following anthracyclinecontaining chemotherapy via peripheral line. Polysorbate 80-free intravenous neurokinin-1 receptor antagonist may provide a safer chemotherapy-induced nausea and vomiting prophylaxis option.
\end{abstract}

Lay abstract: Nausea and vomiting are common side effects of cancer chemotherapy. Fosaprepitant is one of a number of drugs called antiemetics that are used together to prevent this nausea and vomiting. Fosaprepitant is provided in a liquid form, so it can be administered intravenously. However, the liquid also includes polysorbate 80 , which can cause increased sensitivity (hypersensitivity) and side effects at the injection site. These side effects can be serious and sometimes dangerous for patients. In the medical records of 127 patients with cancer who had chemotherapy (anthracycline plus cyclophosphamide) and who had fosaprepitant and other antiemetic drugs to prevent nausea and vomiting, $28 \%$ of patients had increased sensitivity or side effects at the injection site.

First draft submitted: 28 August 2018; Accepted for publication: 20 September 2018; Published online: 10 October 2018

Keywords: chemotherapy-induced nausea and vomiting • fosaprepitant $\bullet$ infusion-site adverse events

Chemotherapy-induced nausea and vomiting (CINV) can profoundly affect patient quality-of-life and cause interruptions and/or dose modifications in chemotherapy regimens [1-3]. Effective antiemetic prophylaxis is an essential component of cancer care, particularly in patients receiving highly emetogenic chemotherapy (HEC) or moderately emetogenic chemotherapy regimens. Consensus antiemetic guidelines generally recommend a combination of a neurokinin-1 (NK-1) receptor antagonist, a 5-hydroxytryptamine type-3 receptor antagonist and dexamethasone, with or without olanzapine, to prevent CINV in these high-risk groups [4-7].

Aprepitant and its intravenous (iv.) prodrug, fosaprepitant, are commonly used NK-1 receptor antagonists. The iv. preparation contains the nonionic surfactant polysorbate 80 to solubilize the fosaprepitant [8]. Polysorbate 80 is a biologically active compound present in a number of iv. formulations, including docetaxel [9] and darbepoetin [10]. Hypersensitivity systemic reactions (HSRs) and infusion-site adverse events (ISAEs) during and after administration of these agents may be partly due to the presence of polysorbate 80 [11-13].

Future Medicine 
ISAEs have been reported to occur in $15-67 \%$ of patients receiving fosaprepitant [14-18]. Furthermore, the incidence of ISAEs may increase when fosaprepitant is administered via a peripheral line or given with anthracyclinecontaining chemotherapy [14,16].

The aim of the current study was to describe the incidence of ISAEs and HSRs associated with the use of fosaprepitant in patients receiving the anthracycline doxorubicin plus cyclophosphamide (AC)-based chemotherapy via a peripheral line in a sample of US cancer centers.

\section{Patients \& methods}

This retrospective chart review was undertaken at 14 sites in the USA, from July through August 2016: ten community-based oncology centers, two hospital-owned community clinics, one community-based satellite office of an academic medical center and one large (nonuniversity) hospital center. Patients were included in the chart review if they were aged 18-80 years, had an Eastern Cooperative Oncology Group performance status of 0 or 1 , were receiving chemotherapy consisting of doxorubicin $\geq 60 \mathrm{mg} / \mathrm{m}^{2}$ and cyclophosphamide $\geq 600 \mathrm{mg} / \mathrm{m}^{2}$ and were treated with fosaprepitant as part of a three-drug antiemetic combination regimen including a 5hydroxytryptamine type- 3 receptor antagonist and dexamethasone. Both the chemotherapy and the antiemetic agents had to be administered via a peripheral iv. line. All inclusion criteria were predefined and patient records were accessed via deidentified Health Insurance Portability and Accountability Act - compliant methodology.

From the deidentified electronic medical records, the occurrence of ISAEs or HSRs was determined using a combination of documented codes, nursing codes and physician notes. Records were searched over multiple chemotherapy cycles for codes relating to ISAEs (pain, erythema, swelling, hives, phlebitis, superficial thrombosis or delayed infusion) or HSRs (flushing, hypotension, bronchospasm and cardiac dysfunction).

Continuous variables (e.g., mean, standard deviation) and categorical variables (e.g., frequency and percentage) were assessed using descriptive statistics.

\section{Results}

The chart review identified 142 patients, aged 28-78 years (mean 55.8 years) with Eastern Cooperative Oncology Group performance status 0 or 1 who were screened for inclusion. Most patients were women (95.1\%), and most patients had breast cancer (91.5\%; Table 1). Of these, 127 patients met all inclusion criteria and were included in the analysis. All 127 patients (100\%) received one cycle of AC-based chemotherapy, 89 (70\%) received two cycles, $51(40 \%)$ received three cycles, $36(28 \%)$ received four cycles and one (1\%) received five cycles.

Among these 127 patients, 35 (27.6\%) had an ISAE and/or an HSR during or after the infusion of the antiemetics and chemotherapy, of whom $19(15 \%)$ had an ISAE only, three (2\%) had an HSR only and $13(10 \%)$ had both an ISAE and an HSR.

ISAEs occurred in 32 patients (25.2\%), alone or in addition to HSRs. These patients had 137 individual ISAEs documented over multiple chemotherapy cycles (range: 0-90 events per cycle). Most of these ISAEs (90 events in 26 patients) occurred in cycle 1. The most common ISAEs were erythema (35 events), pain (34 events) and swelling (25 events) at the infusion site (Table 2).

HSRs occurred in 16 patients (12.6\%), alone or in addition to ISAEs. These patients had 50 individual HSRs documented over multiple chemotherapy cycles (range: 0-36 events per cycle). Most of these HSRs (36 events in 14 patients) occurred in cycle 1 . The most common HSRs were edema/swelling (ten events), erythema (ten events) and dermatitis (nine events; Table 3). No patient developed anaphylaxis.

\section{Discussion}

In this retrospective chart review, $28 \%$ of patients receiving fosaprepitant via a peripheral line for CINV prevention during the administration of AC-based chemotherapy developed an ISAE or HSR during or after the infusion. The proportion of patients who developed at least one ISAE in our study $(25.2 \%)$ is consistent with values reported in two previous chart reviews conducted at single centers in the USA, which found that $34.7 \%$ of patients receiving doxorubicin plus cyclophosphamide [15] and $28.7 \%$ of patients receiving a variety of chemotherapy regimens [16] experienced ISAEs with peripherally administered fosaprepitant. In two retrospective studies in Japan, the proportion of patients experiencing ISAEs was higher with peripherally administered fosaprepitant than in our study: $41.7 \%$ of patients who received anthracycline- or cisplatin-based chemotherapy [14] and $66.7 \%$ of patients who received anthracycline-based chemotherapy [18] reported ISAEs, although the latter study had a small sample size $(\mathrm{n}=24)$. In contrast, in a Japanese randomized, double-blind, placebo-controlled Phase III trial of 
Table 1. Patient demographics and baseline clinical characteristics.

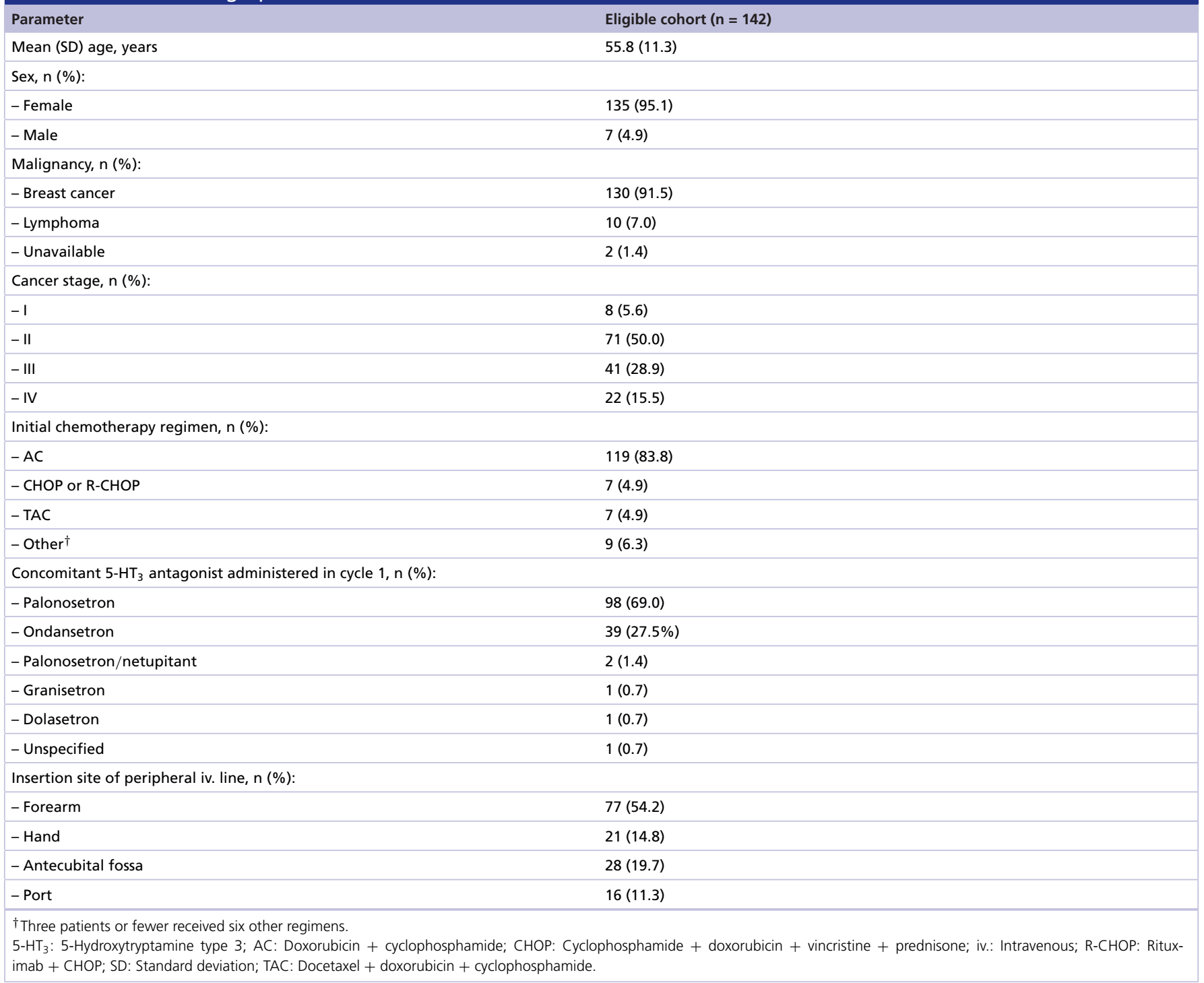

fosaprepitant in patients receiving cisplatin-based chemotherapy, ISAEs were reported in $23.6 \%$ of patients [17]. Overall, the proportion of patients experiencing ISAEs in a real-world setting appears to be much higher than that in the prescribing information for fosaprepitant, which reports ISAEs in 3\% of patients receiving HEC and in $2.2 \%$ receiving moderately emetogenic chemotherapy [8], and even these values may underestimate the incidence of ISAEs, as these events are only captured if reported.

The incidence of ISAEs and/or HSRs associated with chemotherapy may be influenced by a range of factors, including route of administration, infusion rate, location of iv. line, formulation excipients, concomitant medications, prior exposure to treatments, patient age and co-morbidities [16,19]. Polysorbate 80 has been implicated in a number of systemic reactions including hypersensitivity, as well as injection- or infusion-site pain, erythema and thrombophlebitis [11-13]. These events are probably histamine-mediated, based on in vitro and in vivo data on the effects of polysorbate 80 [20]. In addition, doxorubicin is a vesicant and also stimulates histamine release [21], which may explain why the combination of a polysorbate 80-containing antiemetic and an anthracycline may be associated with a particularly high incidence of ISAEs and HSRs [14,18,22]. Factors associated with an increased risk of ISAEs in patients receiving fosaprepitant and anthracyclines are age, insertion of the peripheral line into the hand (rather than the forearm or antecubital fossa), and slow infusion of fluids $(<100 \mathrm{ml} / \mathrm{h})$ [16]. 


\begin{tabular}{|c|c|c|c|c|c|c|}
\hline ISAES & Cycle 1 & Cycle 2 & Cycle 3 & Cycle 4 & Cycle 5 & $\begin{array}{l}\text { Total incidence per } \\
\text { reaction }\end{array}$ \\
\hline Total number of patients & 127 & 89 & 51 & 36 & 1 & - \\
\hline Unique patients with ISAE, $n^{\dagger}$ & 26 & 15 & 1 & 2 & 0 & - \\
\hline Erythema at site & 24 & 10 & 0 & 1 & 0 & 35 \\
\hline Pain at site & 24 & 9 & 0 & 1 & 0 & 34 \\
\hline Swelling at site & 18 & 6 & 0 & 1 & 0 & 25 \\
\hline Vein discoloration at site & 8 & 5 & 1 & 2 & 0 & 16 \\
\hline $\begin{array}{l}\text { Venous engorgement, hardening or } \\
\text { induration }\end{array}$ & 4 & 3 & 0 & 1 & 0 & 8 \\
\hline Superficial thrombosis at site & 2 & 2 & 0 & 1 & 0 & 5 \\
\hline Infusion-site hives & 4 & 0 & 0 & 0 & 0 & 4 \\
\hline Extravasation at site & 2 & 2 & 0 & 0 & 0 & 4 \\
\hline Superficial thrombophlebitis at site & 1 & 1 & 0 & 1 & 0 & 3 \\
\hline Thrombosis at site & 3 & 0 & 0 & 0 & 0 & 3 \\
\hline Deep vein thrombosis & 0 & 0 & 0 & 0 & 0 & 0 \\
\hline Other & 0 & 0 & 0 & 0 & 0 & 0 \\
\hline Total ISAEs per cycle & 90 & 38 & 1 & 8 & 0 & \\
\hline
\end{tabular}

\begin{tabular}{|c|c|c|c|c|c|c|}
\hline HSRs & Cycle 1 & Cycle 2 & Cycle 3 & Cycle 4 & Cycle 5 & $\begin{array}{l}\text { Total incidence per } \\
\text { reaction }\end{array}$ \\
\hline Total number of patients & 127 & 89 & 51 & 36 & 1 & - \\
\hline Unique patients with $H S R, n^{\dagger}$ & 14 & 6 & 3 & 0 & 0 & - \\
\hline Edema/swelling & 9 & 1 & 0 & 0 & 0 & 10 \\
\hline Erythema & 6 & 2 & 2 & 0 & 0 & 10 \\
\hline Dermatitis & 6 & 3 & 0 & 0 & 0 & 9 \\
\hline Rash & 6 & 0 & 0 & 0 & 0 & 6 \\
\hline Flushing & 2 & 1 & 2 & 0 & 0 & 5 \\
\hline Dyspnea & 2 & 0 & 0 & 0 & 0 & 2 \\
\hline Bronchospasm/shortness of breath & 2 & 0 & 0 & 0 & 0 & 2 \\
\hline Hypotension/hypertension & 0 & 1 & 0 & 0 & 0 & 1 \\
\hline Chills/fever & 1 & 0 & 0 & 0 & 0 & 1 \\
\hline Coughing & 0 & 1 & 0 & 0 & 0 & 1 \\
\hline Vomiting at time of infusion & 1 & 0 & 0 & 0 & 0 & 1 \\
\hline Other & 1 & 0 & 1 & 0 & 0 & 2 \\
\hline Total HSRs per cycle & 36 & 9 & 5 & 0 & 0 & - \\
\hline
\end{tabular}

Our data are consistent with other reports that the rate of ISAEs and/or HSRs with anthracycline-based chemotherapy is highest during the first cycle of chemotherapy [23]. Most of these reactions are grade 1 or 2 events $[16,18,23]$, and routine use of premedication is not indicated [23,24]. Most will resolve within hours, but approximately one in four will take days or even weeks to resolve [16]. Current recommendations for managing nonurgent ISAEs or HSRs include slowing the anthracycline infusion rate [23] and instituting supportive care (e.g., applying warmth to the affected area) [16,24]. Acute breathing or circulatory symptoms require urgent intervention [23,24].

Some institutions have changed their clinical practice as a result of ISAEs associated with fosaprepitant. A retrospective chart review at the James Cancer Center (OH, USA) showed ISAEs in 15\% of patients who received 
fosaprepitant via a peripheral iv. line, all of which were grade 2 or less. Multivariate analyses found that risk factors for ISAEs were increased age, location of iv. line (hand vs forearm or antecubital fossa) and simultaneous maintenance iv. fluid rate less than $100 \mathrm{ml} / \mathrm{h}$. Subsequently, the protocol at that site was amended to include strategies to prevent or reduce the incidence of ISAEs. Those strategies included switching from fosaprepitant to the orally administered aprepitant or switching to another antiemetic regimen that excluded fosaprepitant. Alternatively, if fosaprepitant was used, it was diluted from 1 to $0.6 \mathrm{mg} / \mathrm{ml}$ (i.e., diluted in $250 \mathrm{ml}$ of $0.9 \%$ sodium chloride base solution instead of $150 \mathrm{ml}$ ) and the administration time extended from 20 to $30 \mathrm{~min}$ [16].

After the inclusion of fosaprepitant in the Mayo Clinic (MN, USA) antiemetic guidelines in 2011 for the prevention of CINV in patients receiving HEC, a retrospective chart review showed a significant increase in the incidence of ISAEs in patients receiving AC-based chemotherapy compared with those receiving non-anthracycline HEC regimens (odds ratio: 8.1; 95\% CI: 2.0-31.9) [22]. Another retrospective chart review in patients receiving AC-based chemotherapy at the same institution found an increased incidence of ISAEs in patients receiving fosaprepitant (34.7\%) compared with those receiving the orally administered aprepitant (2.3\%). All ISAEs were associated with the use of a peripheral iv. line for fosaprepitant administration [15]. As a result of these findings, the Mayo Clinic antiemetic guidelines were changed in 2015 to recommend the use of aprepitant, rather than fosaprepitant, in patients receiving AC-based chemotherapy.

If fosaprepitant is to be used as part of an antiemetic regimen for patients receiving AC-based chemotherapy, using a central line may be preferable, as the incidence of ISAEs and HSRs appears to be lower using central rather than peripheral venous access $[15,22]$.

Limitations of our study include its retrospective nature with the inherent challenges of interpreting medical records with regard to ISAEs, and that the severity and resolution of ISAEs and HSRs were not measured. In addition, the patient population was primarily women with breast cancer receiving AC chemotherapy, so the findings may not be generalizable to a wider patient population. However, in a study with a patient population of $74 \%$ men and $71 \%$ with lung cancer, a higher percentage of ISAEs was observed in patients receiving fosaprepitant versus placebo (23.6 vs $12.4 \%)$ [17].

\section{Conclusion}

The current formulation of fosaprepitant, which contains polysorbate 80 , is associated with a high rate of ISAEs and/or HSRs in patients receiving anthracycline-containing chemotherapy through a peripheral line. These events are generally mild and most likely to occur during the first infusion. Nevertheless, an iv. formulation of fosaprepitant that does not contain polysorbate 80 would be a useful alternative to the current formulation when patients require a combination antiemetic regimen for CINV prophylaxis, and it may provide a safer treatment option.

\section{Summary points}

- Chemotherapy-induced nausea and vomiting (CINV) may affect quality of life and lead to disruptions in chemotherapy for patients with cancer.

- Consensus antiemetic guidelines recommend a three- or four-drug regimen for CINV prevention, which includes aprepitant and its intravenous (iv.) prodrug, fosaprepitant, which are commonly used neurokinin-1 receptor antagonists.

- iv. fosaprepitant contains the nonionic surfactant polysorbate 80 , which may be responsible for hypersensitivity systemic reactions and infusion-site adverse events occurring during or after administration of the drug.

- A retrospective chart review of 127 patients receiving fosaprepitant via a peripheral line revealed that 35 (28\%) patients developed an infusion-site adverse event and/or hypersensitivity systemic reaction during or after infusion of antiemetics and chemotherapy.

- An iv. formulation of fosaprepitant that is free of polysorbate 80 may be a safer treatment option for CINV prophylaxis.

\section{Acknowledgements}

Research support was provided by Heron Therapeutics, CA, USA. Medical writing assistance was provided by YE Yarker of SciStrategy Communications (PA, USA), supported by Heron Therapeutics. 
Financial \& competing interests disclosure

R Boccia reports employment by and leadership role at Center for Cancer and Blood Disorders; has been a consultant/advisor for Gilead, Heron Therapeutics, Inc. and Incyte; has been part of a speakers bureau and received honoraria and compensation for travel and accommodations from AbbVie, Amgen, AstraZeneca, BMS, Celgene, DSI, Genentech, Gilead, Heron Therapeutics and Incyte; and his practice has received research funding from Incyte. RB Geller, N Clendeninn and T Ottoboni report employment by and stock ownership in Heron Therapeutics, Inc. N Clendeninn and T Ottoboni report a leadership role at Heron Therapeutics, Inc. The authors have no other relevant affiliations or financial involvement with any organization or entity with a financial interest in or financial conflict with the subject matter or materials discussed in the manuscript apart from those disclosed.

Writing assistance was utilised in this manuscript.

Ethical conduct of research

This was a retrospective longitudinal analysis from a de-identified HIPAA compliant claims database, so no institutional review board approval was necessary.

\section{Open access}

This work is licensed under the Attribution-NonCommercial-NoDerivatives 4.0 Unported License. To view a copy of this license, visit http://creativecommons.org/licenses/by-nc-nd/4.0/

\section{References}

Papers of special note have been highlighted as: $\bullet$ of interest or $\bullet \bullet$ of considerable interest

1. Hassan BA, Yusoff ZB. Negative impact of chemotherapy on breast cancer patients QOL - utility of antiemetic treatment guidelines and the role of race. Asian Pac. J. Cancer Prev. 11(6), 1523-1527 (2010).

2. Hesketh PJ. Chemotherapy-induced nausea and vomiting. N. Engl. J. Med. 358(23), 2482-2494 (2008).

3. Kim HK, Hsieh R, Chan A et al. Impact of CINV in earlier cycles on CINV and chemotherapy regimen modification in subsequent cycles in Asia Pacific clinical practice. Support. Care Cancer 23(1), 293-300 (2015).

4. NCCN Clinical Practice Guidelines in Oncology: Antiemesis - Version 3.2018. (2018). www.nccn.org/professionals/physician_gls/default.aspx

- NCCN antiemesis guidelines for the prevention of chemotherapy-induced nausea and vomiting (CINV).

5. Herrstedt J, Roila F, Warr D et al. 2016 Updated MASCC/ESMO consensus recommendations: prevention of nausea and vomiting following high emetic risk chemotherapy. Support Care Cancer 25(1), 277-288 (2017).

- MASCC/ESMO antiemesis guidelines for the prevention of CINV following highly emetogenic chemotherapy.

6. Hesketh PJ, Kris MG, Basch E et al. Antiemetics: American Society of Clinical Oncology Clinical Practice Guideline update. J. Clin. Oncol. JCO2017744789 (2017).

- American Society of Clinical Oncology antiemesis guidelines for the prevention of CINV.

7. Roila F, Warr D, Hesketh PJ et al. 2016 updated MASCC/ESMO consensus recommendations: prevention of nausea and vomiting following moderately emetogenic chemotherapy. Support Care Cancer 25(1), 289-294 (2017).

- MASCC/ESMO guidelines for the prevention of CINV following moderately emetogenic chemotherapy.

8. Emend (fosaprepitant) for injection [prescribing information]. Merck Sharp \& Dohme Corp., Whitehouse Station, NY, USA (2017).

9. Taxotere (docetaxel) injection concentrate, intravenous infusion [prescribing information]. Sanofi-Aventis, Bridgewater, NJ, USA (2015).

10. Aranesp (darbepoetin alfa) injection, for intravenous or subcutaneous use [prescribing information]. Amgen Inc., Thousand Oaks, CA, USA (2017).

11. Coors EA, Seybold H, Merk HF, Mahler V. Polysorbate 80 in medical products and nonimmunologic anaphylactoid reactions. Ann. Allergy Asthma Immunol. 95(6), 593-599 (2005).

-. Review of polysorbate 80 as a solubilizing agent and associated hypersensitivity reactions.

12. Steele RH, Limaye S, Cleland B, Chow J, Suranyi MG. Hypersensitivity reactions to the polysorbate contained in recombinant erythropoietin and darbepoietin. Nephrology (Carlton) 10(3), 317-320 (2005).

13. Ten Tije AJ, Verweij J, Loos WJ, Sparreboom A. Pharmacological effects of formulation vehicles: implications for cancer chemotherapy. Clin. Pharmacokinet. 42(7), 665-685 (2003).

14. Fujii T, Nishimura N, Urayama KY et al. Differential impact of fosaprepitant on infusion site adverse events between cisplatin- and anthracycline-based chemotherapy regimens. Anticancer Res. 35(1), 379-383 (2015).

15. Leal AD, Kadakia KC, Looker $S$ et al. Fosaprepitant-induced phlebitis: a focus on patients receiving doxorubicin/cyclophosphamide therapy. Support. Care Cancer 22(5), 1313-1317 (2014). 
-• Retrospective study of infusion-site adverse events with fosaprepitant administered via a peripheral line in patients receiving anthracycline plus cyclophosphamide chemotherapy.

16. Lundberg JD, Crawford BS, Phillips G, Berger MJ, Wesolowski R. Incidence of infusion-site reactions associated with peripheral intravenous administration of fosaprepitant. Support. Care Cancer 22(6), 1461-1466 (2014).

-. Retrospective study of infusion-site adverse events with fosaprepitant administered via a peripheral line.

17. Saito H, Yoshizawa H, Yoshimori K et al. Efficacy and safety of single-dose fosaprepitant in the prevention of chemotherapy-induced nausea and vomiting in patients receiving high-dose cisplatin: a multicentre, randomised, double-blind, placebo-controlled Phase 3 trial. Ann. Oncol. 24(4), 1067-1073 (2013).

18. Sato $Y$, Kondo M, Inagaki A et al. Highly frequent and enhanced injection site reaction induced by peripheral venous injection of fosaprepitant in anthracycline-treated patients. J. Cancer 5(5), 390-397 (2014).

19. Shepherd GM. Hypersensitivity reactions to chemotherapeutic drugs. Clin. Rev. Allergy Immunol. 24(3), 253-262 (2003).

20. Masini E, Planchenault J, Pezziardi F, Gautier P, Gagnol JP. Histamine-releasing properties of polysorbate 80 in vitro and in vivo: correlation with its hypotensive action in the dog. Agents Actions 16(6), 470-477 (1985).

21. Bristow MR, Sageman WS, Scott RH et al. Acute and chronic cardiovascular effects of doxorubicin in the dog: the cardiovascular pharmacology of drug-induced histamine release. J. Cardiovasc. Pharmacol. 2(5), 487-515 (1980).

22. Hegerova LT, Leal AD, Grendahl DC et al. An analysis of fosaprepitant-induced venous toxicity in patients receiving highly emetogenic chemotherapy. Support. Care Cancer 23(1), 55-59 (2015).

23. Rosello S, Blasco I, Garcia Fabregat L, Cervantes A, Jordan K. Management of infusion reactions to systemic anticancer therapy: ESMO Clinical Practice Guidelines. Ann. Oncol. 28(Suppl. 4), iv100-iv118 (2017).

24. Joerger M. Prevention and handling of acute allergic and infusion reactions in oncology. Ann. Oncol. 23(Suppl. 10), x313-x319 (2012). 
